\title{
La motivation en formation des professionnels de la santé
}

\author{
Guide AMEE \\ Motivation in medical education
}

\author{
Thierry PELACCIA ${ }^{1,2}$ et Rolland VIAU ${ }^{3}$ \\ 1 Faculté de médecine, Centre de formation et de recherche en pédagogie des sciences de la santé (CFRPS), \\ Université de Strasbourg, Strasbourg, France \\ 2 Service d'aide médicale urgente (SAMU 67), Hôpitaux universitaires de Strasbourg, Strasbourg, France ; \\ 3 Faculté d'éducation, Université de Sherbrooke, Sherbrooke, Canada
}

* Cet article est la traduction française d'un travail original élaboré en tant que guide de l'Association for Medical Education in Europe, qui a fait l'objet d'une publication princeps en anglais (Pelaccia T, Viau R. Motivation in medical education. Med Teach 2017;39:136-140) qui constitue elle-même une partie de l'AMEE Guide N 115, disponible sur : www.amee.org.) Sa publication s'inscrit dans le cadre d'un partenariat institutionnel entre l'Association for Medical Education in Europe (AMEE) et la Société internationale francophone d'éducation médicale (SIFEM), et leurs organes officiels d'expression respectifs, les revues Medical Teacher et Pédagogie Médicale. Une première version de la traduction en français de l'article princeps a été proposée par les auteurs, Thierry Pelaccia (Université de Strasbourg) et Rolland Viau (Université de Sherbrooke). La version finale résulte d'un processus de révision collégiale conduit au sein du comité de rédaction de la revue Pédagogie Médicale, coordonné par Jean Jouquan.

Choix éditorial : l'article original publié dans la revue Medical Teacher fait référence à la motivation chez les étudiants en médecine. Cependant, tous les éléments discutés dans ce guide, et notamment les stratégies motivationnelles qui sont exposées, s'appliquent à l'ensemble des étudiants en formation professionnelle en santé. En conséquence, dans la présente version française, en cohérence avec la dimension interprofessionnelle des activités liées au projet institutionnel de la SIFEM, le choix a été fait de traduire la locution anglaise medical education d'une manière générique, en faisant référence, selon le contexte, à la notion d'éducation en sciences de la santé et/ou pour les professionnels de la santé.

\author{
Mots-clés \\ motivation; perception \\ de la valeur; \\ sentiment d'efficacité \\ personnelle ; \\ perception de \\ contrôlabilité ; \\ environnement \\ d'apprentissage ; \\ stratégies \\ d'enseignement
}

Résumé - Problématique : La motivation est un concept qui fascine les chercheurs depuis de nombreuses décennies. Le milieu de l'éducation des professionnels de la santé s'y est intéressé plus récemment, en questionnant le postulat implicitement admis jusqu'alors, selon lequel les étudiants en médecine ou plus généralement en santé sont forcément motivés, en raison de leur engagement dans une formation très professionnalisante débouchant sur un métier bien spécifique. En effet, la motivation est un déterminant majeur de la qualité des apprentissages et de la réussite. Un défaut de motivation peut expliquer bien des situations de découragement, de désintérêt ou d'abandon, que les enseignants constatent parfois chez les étudiants en médecine ou en santé, avec un sentiment d'impuissance ou de résignation. Exégèse: Après avoir décrit l'importance de la motivation pour l'apprentissage lié à la formation en santé, ce guide définit 
le concept de motivation en l'ancrant dans une approche sociocognitive. Dans une deuxième partie, nous formulons un certain nombre de recommandations basées sur le «modèle de la dynamique motivationnelle», qui offre une multitude et une variété de leviers afin d'agir positivement sur la motivation des étudiants.

Keywords motivation ; perception of the value of an educational activity ; perceived self-efficacy ; perception of controllability ; learning environment; teaching strategies

\begin{abstract}
Background: Motivation is a concept which has fascinated researchers for many decades. The field of medical education has become interested in motivation recently, having always assumed that medical students must be motivated because of their commitment to highly specific training, leading to a very specific profession. However, motivation is a major determinant of the quality of learning and success, the lack of which may well explain why teachers sometimes observe medical students who are discouraged, have lost interest or abandon their studies, with a feeling of powerlessness or resignation. Analysis: After describing the importance of motivation for learning in medicine, this Guide will define the concept of motivation, setting it within the context of a social cognitive approach. In the second part of this Guide, recommendations are made, based upon the so-called "motivational dynamic model", which provides a multitude of various strategies with positive effects on students' motivation to learn.
\end{abstract}

\section{Points pratiques}

- Un étudiant motivé mobilise des stratégies d'apprentissage plus efficaces, persévère en cas de difficultés ou d'échecs, et atteint un niveau de performance plus élevé qu'un étudiant peu motivé.

- Les étudiants en médecine ou en santé étant le plus souvent très motivés pour obtenir leur diplôme, les enjeux, pour les enseignants, consistent à agir positivement sur la motivation à apprendre.

- La motivation à apprendre est d'autant plus élevée que les étudiants perçoivent un intérêt ou une utilité dans les activités que les enseignants leur demandent de faire (perception de la valeur), qu'ils se sentent capables de réaliser les activités en question (sentiment d'efficacité personnelle) et qu'ils ont le sentiment d'avoir leur mot à dire sur le déroulement de ces activités (perception de contrôlabilité).

- Le fait d'explorer les attentes et les projets des étudiants, d'expliquer à quoi sert la matière enseignée, d'associer les activités pédagogiques à un défi, de problématiser les apprentissages et de renforcer les liens entre la théorie et la pratique sont des stratégies qui permettent d'agir positivement sur la perception de la valeur de l'activité.
- Le fait de favoriser la réussite, de promouvoir les évaluations motivantes, de prendre en compte l'effet du groupe, d'offrir un feed-back bienveillant et de donner aux étudiants l'opportunité d'endosser des fonctions d'enseignement sont des stratégies qui permettent d'agir positivement sur le sentiment d'efficacité personnelle.

- Le fait de laisser aux étudiants la responsabilité de choix signifiants est une stratégie qui permet d'agir positivement sur la perception de contrôlabilité.

\section{Introduction}

La motivation fait partie de ces concepts liés à l'éducation, dont se sont emparés à la fois le grand public - tout le monde a un avis sur la motivation, souvent fondé sur des événements et des expériences passés - et, depuis la fin du dix-neuvième siècle, les chercheurs du champ de la psychologie et de l'éducation. Le milieu de l'enseignement supérieur s'est intéressé plus tardivement à la motivation. Pendant longtemps, il a en effet été admis que les étudiants à ce niveau étaient forcément motivés, notamment dans les filières hautement professionnalisantes comme celles des sciences de la santé. De ce fait, les 
études spécifiquement réalisées sur la motivation en formation des sciences de la santé sont rares, même si leur nombre s'est accru depuis le début des années deux mille ${ }^{[1]}$.

La plupart de ces études ont montré que la motivation était associée à des effets positifs sur le plan de l'apprentissage ${ }^{[2-6]}$. Plus précisément, plus le niveau de motivation d'un étudiant engagé dans une formation professionnelle en santé est élevé, meilleure est la qualité de ses apprentissages et des stratégies d'apprentissage qu'il utilise, et plus il persévère et atteint des niveaux élevés de performance. Les recherches menées dans le domaine de la motivation ont également permis de conclure que bien que les enseignants ne peuvent à eux seuls être considérés comme responsables de la motivation à apprendre de leurs étudiants, leur influence est très importante ${ }^{[7]}$. Ces résultats légitiment la pertinence, pour les enseignants des formations en santé, de prendre en compte la dimension motivationnelle de l'apprentissage et de favoriser la mise en œuvre de stratégies de formation et d'évaluation qui permettent d'agir positivement sur la motivation à apprendre de leurs étudiants.

Les étudiants de l'enseignement supérieur sont en effet principalement motivés pour réussir, particulièrement dans les facultés de médecine, où le processus de sélection favorise ce type de motivation, plutôt que la motivation à apprendre ${ }^{[8]}$. Ainsi, le principal enjeu consiste à motiver les étudiants à s'impliquer en profondeur dans leur apprentissage et à persévérer en utilisant des stratégies de niveau taxonomique élevé. L'objectif de ce guide est de vous fournir, en tant qu'enseignants en sciences de la santé, des outils vous permettant d'agir positivement sur la motivation à apprendre de vos étudiants, en mobilisant des stratégies d'enseignement et d'évaluation motivantes. Pour atteindre cet objectif, nous devons dans un premier temps opérationnaliser le concept de «motivation à apprendre», en le définissant.

\section{Qu'est-ce que la motivation?}

De façon très générale, Graham \& Weiner considèrent que «la motivation consiste à étudier pourquoi les gens pensent et se comportement comme ils le font ${ }^{[9]}$. Il n'existe cependant pas de définition consensuelle de la motivation, au regard des dizaines de théories qui ont été élaborées autour de ce concept. Parmi elles, l'approche sociocognitive a pris une importance considérable, car elle est considérée comme une approche hautement intégrative et holistique afin d'appréhender le concept de motivation à apprendre ${ }^{[10]}$. Selon cette approche, la motivation à apprendre est déterminée à la fois pas l'individu luimême et par son environnement. Plus précisément, elle est le fruit d'une interaction constante entre les perceptions d'un étudiant, ses comportements d'apprentissage et des facteurs environnemen$\operatorname{taux}^{[7,11]}$.

L'approche sociocognitive permet de comprendre que la motivation ne renvoie pas uniquement au choix d'entreprendre telle ou telle activité, mais également à la direction, à l'intensité et à la persistance du comportement d'une personne une fois que son choix a été fait. Elle permet également de souligner le fait que la motivation varie en fonction $\mathrm{du}$ moment, du contexte et du domaine au sein duquel elle est étudiée. Il ne s'agit donc ni d'un trait de personnalité ni d'un état statique ${ }^{[12,13]}$. C'est la raison pour laquelle Viau préfère au terme de motivation celui de «dynamique motivationnelle» ${ }^{[7]}$.

Ces considérations nous conduisent à retenir la définition suivante de la motivation : «[la motivation est] un phénomène qui tire sa source dans des perceptions a de lui-même et de son environnement, et qui a pour conséquence qu'il choisit de s'engager à accomplir l'activité pédagogique qu'on lui propose et de persévérer dans son accomplissement, et ce, dans le but d'apprendre» ${ }^{[7]}$.

Afin d'aider les enseignants à prendre en compte la motivation dans leurs stratégies d'enseignement et d'évaluation, et d'agir positivement sur la motivation à apprendre de leurs étudiants, nous ancrerons nos propositions autour du «modèle de la dynamique motivationnelle», qui possède une forte valeur instrumentale et qui offre une multitude de leviers d'action susceptibles d'être utilisés par un enseignant dans le but d'agir sur la motivation à apprendre de ses étudiants. 


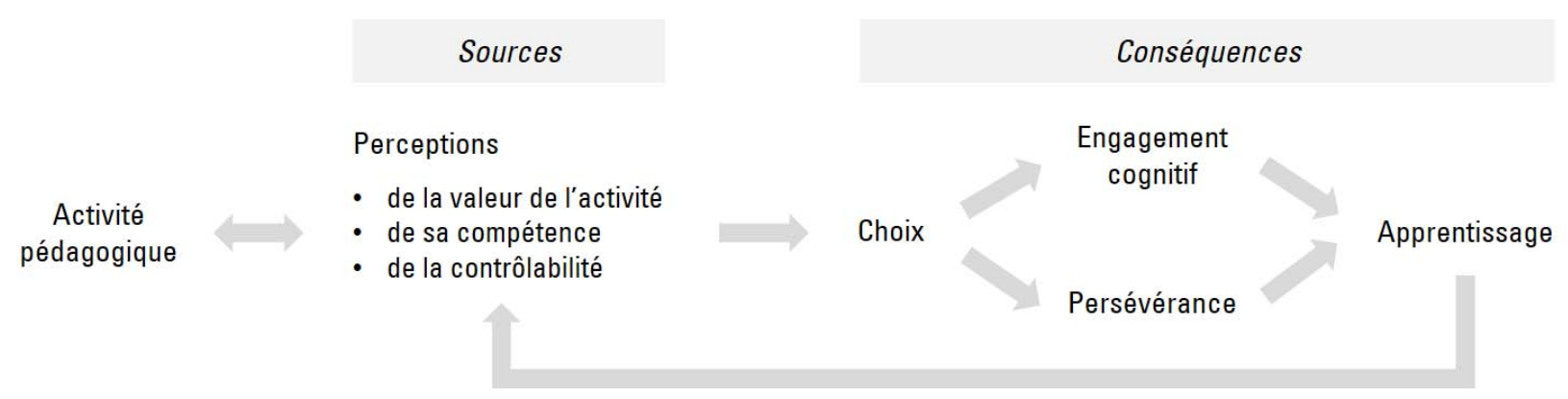

Fig. 1. Représentation schématisée du modèle de la dynamique motivationnelle. Figure extraite de Viau ${ }^{[7]}$ et reproduite avec l'aimable autorisation des Éditions du Renouveau Pédagogique.

\section{Le modèle de la dynamique motivationnelle}

Le modèle de la dynamique motivationnelle met en relation des sources intrinsèques de la motivation et leurs conséquences sur le plan de la qualité des apprentissages, tel que l'illustre la figure 1 .

La dynamique motivationnelle d'un étudiant à apprendre se forge autour de trois perceptions :

- la perception de la valeur d'une activité pédagogique (également dénommée «valeur subjective de la tâche») est définie comme le jugement qu'un étudiant porte sur l'intérêt et l'utilité d'accomplir une activité qui lui est proposée, et ce, en fonction des buts qu'il poursuit ${ }^{[7,14]}$. Plus les étudiants accordent de valeur aux activités pédagogiques qui leur sont proposées, plus ils persévèrent et s'y engagent en profondeur ${ }^{[15,16]}$.

- le sentiment d'efficacité personnelle (également dénommé «perception de compétence») est le jugement que l'étudiant porte sur sa capacité à réussir de manière adéquate l'activité pédagogique qui lui est proposée ${ }^{[7]}$. Plus un étudiant perçoit un sentiment d'efficacité personnelle élevé, plus il se fixe des objectifs élevés, choisit des activités qui suscitent chez lui un défi, régule ses efforts, persévère, gère son stress et son anxiété, et par conséquent, atteint des niveaux élevés de performance.

- la perception de la contrôlabilité (également dénommée «contrôlé perçu») est définie comme étant le degré de contrôle qu'un étudiant croit exercer sur le déroulement d'une activité ${ }^{[7]}$. Un étudiant a une perception de contrôlabilité élevée s'il juge qu'il a son mot à dire sur la façon dont se déroule l'activité pédagogique qui lui est proposée. Les étudiants dont la perception de contrôlabilité est élevée sont plus engagés et persévèrent davantage dans leur apprentissage ${ }^{[17]}$.

Selon le modèle de la dynamique motivationnelle, un «étudiant motivé» juge utiles ou intéressantes les activités pédagogiques qui lui sont proposées (perception de la valeur de l'activité pédagogique), se sent capable d'accomplir ces activités comme il le souhaite (sentiment d'efficacité personnelle) et a l'impression qu'on le responsabilise par rapport au déroulement de ses apprentissages (perception de contrôlabilité). Nous allons maintenant discuter des stratégies qui vous permettront d'agir positivement sur ces trois perceptions, c'est-à-dire sur la motivation à apprendre de vos étudiants.

\section{Comment agir positivement sur la motivation à apprendre de vos étudiants?}

En tant qu'enseignant, il est possible que vous soyez déjà familier avec un certain nombre de stratégies dont nous discuterons ci-dessous et qui sont résumées dans le tableau I, car beaucoup sont des stratégies d'enseignement qui visent à agir positivement sur la qualité des apprentissages. Le modèle de la dynamique motivationnelle vous aidera à mieux 
Tableau I. Différents stratégies et principes de mise en œuvre permettant d'agir positivement sur les trois sources de la dynamique motivationnelle.

\begin{tabular}{|c|c|c|}
\hline $\begin{array}{c}\text { Source de la } \\
\text { dynamique } \\
\text { motivationnelle } \\
\text { principalement ciblée }\end{array}$ & Stratégie & Mise en ouvre pratique \\
\hline \multirow{5}{*}{$\begin{array}{l}\text { Perception de la valeur } \\
\text { de l'activité }\end{array}$} & $\begin{array}{l}\text { Explorez les attentes et les } \\
\text { projets des étudiants }\end{array}$ & $\begin{array}{l}\text { - tour de table } \\
\text { - entretien individuel } \\
\text { - entretien collectif } \\
\text { - questionnaire écrit }\end{array}$ \\
\hline & $\begin{array}{l}\text { Expliquez à quoi servira la } \\
\text { matière enseignée }\end{array}$ & $\begin{array}{l}\text { - exposer les objectifs d'apprentissage } \\
\text { - lier les objectifs d'apprentissage à la pratique professionnelle } \\
\text { future des étudiants } \\
\text { - lier l'apprentissage des sciences biomédicales à celui des } \\
\text { sciences cliniques, par exemple, en associant lors de la } \\
\text { préparation et de la mise en œuvre du cours un fondamentaliste } \\
\text { et un clinicien } \\
\text { - favoriser précocement le contact des étudiants avec les patients }\end{array}$ \\
\hline & $\begin{array}{l}\text { Associez la réalisation de } \\
\text { l'activité à un défi }\end{array}$ & $\begin{array}{l}\text { - définir des objectifs d'apprentissage de complexité moyenne, } \\
\text { ni trop simples, ni trop difficiles à atteindre }\end{array}$ \\
\hline & $\begin{array}{l}\text { Problématisez les } \\
\text { apprentissages }\end{array}$ & $\begin{array}{l}\text { - créer des liens entre les sciences biomédicales et les sciences } \\
\text { cliniques } \\
\text { - associer les tâches d'apprentissage à la nécessiter de résoudre } \\
\text { un problème } \\
\text { - commencer la séquence d'apprentissage par une vignette } \\
\text { clinique, une anecdote ou des ressources iconographiques } \\
\text { - privilégier l'évaluation en contexte authentique }\end{array}$ \\
\hline & $\begin{array}{l}\text { Renforcez les liens entre la } \\
\text { théorie et la pratique }\end{array}$ & $\begin{array}{l}\text { - créer des expériences de soins en amont (ou simultanément) } \\
\text { par rapport aux apprentissages théoriques }\end{array}$ \\
\hline \multirow[t]{2}{*}{$\begin{array}{l}\text { Sentiment d'efficacité } \\
\text { personnelle }\end{array}$} & $\begin{array}{l}\text { Favorisez la réussite et } \\
\text { promouvez les évaluations } \\
\text { motivantes }\end{array}$ & $\begin{array}{l}\text { - accompagner l'échec } \\
\text { - ne pas utiliser la note comme seul indicateur de sanction ou de } \\
\text { réussite } \\
\text { - prendre le temps de souligner les acquis avant de relever les } \\
\text { erreurs } \\
\text { - rassurer de façon réaliste l'étudiant sur le fait qu'il est capable } \\
\text { de réussir } \\
\text { - valoriser les progrès et la persévérance } \\
\text { - rendre explicites les critères d'évaluation } \\
\text { - ne pas multiplier les évaluations donnant lieu à la délivrance } \\
\text { d'une note }\end{array}$ \\
\hline & $\begin{array}{l}\text { Prenez en compte les } \\
\text { conséquences de } \\
\text { l'expérience vicariante }\end{array}$ & $\begin{array}{l}\text { - offrir un feed-back destiné également aux observateurs d'une } \\
\text { tâche d'apprentissage, en particulier lorsque celle-ci n'a pas été } \\
\text { «réussie» par le pair observé }\end{array}$ \\
\hline
\end{tabular}


Tableau I. Suite.

\begin{tabular}{|c|c|c|}
\hline $\begin{array}{c}\text { Source de la } \\
\text { dynamique } \\
\text { motivationnelle } \\
\text { principalement ciblée }\end{array}$ & Stratégie & Mise en ouvre pratique \\
\hline \multirow[t]{2}{*}{$\begin{array}{l}\text { Sentiment d'efficacité } \\
\text { personnelle }\end{array}$} & $\begin{array}{l}\text { Offrez un feed-back } \\
\text { bienveillant }\end{array}$ & $\begin{array}{l}\text { - considérer les feed-back informels comme importants pour la } \\
\text { motivation de l'étudiant } \\
\text { - ne pas cibler le feed-back sur la seule sanction des erreurs } \\
\text { - valoriser les aspects positifs de la réalisation d'une activité } \\
\text { - offrir aux étudiants un feed-back ciblé sur les questions d'ap- } \\
\text { prentissage et non sur leur personne } \\
\text { - utiliser une sémantique motivante, axée sur les opportunités de } \\
\text { progression }\end{array}$ \\
\hline & $\begin{array}{l}\text { Permettez aux étudiants } \\
\text { d'endosser des fonctions } \\
\text { d'enseignement }\end{array}$ & $\begin{array}{l}\text { - donner aux étudiants en apprentissage par problèmes l'oppor- } \\
\text { tunité d'expliquer à leurs pairs les contenus dont ils sont acquis } \\
\text { la maîtrise } \\
\text { - favoriser l'implantation de dispositifs de tutorat des étudiants } \\
\text { par les étudiants des années supérieures }\end{array}$ \\
\hline $\begin{array}{l}\text { Perception de } \\
\text { contrôlabilité }\end{array}$ & $\begin{array}{l}\text { Laissez aux étudiants des } \\
\text { opportunités d'effectuer } \\
\text { des choix signifiants pour } \\
\text { eux }\end{array}$ & $\begin{array}{l}\text { - laisser choisir un stage } \\
\text { - laisser choisir une unité d'enseignement } \\
\text { - laisser choisir une modalité d'évaluation, etc. }\end{array}$ \\
\hline
\end{tabular}

comprendre pourquoi ces stratégies ont également un effet positif sur la motivation à apprendre des étudiants en médecine ou en santé. Bien entendu, vous devrez décliner ces propositions selon le milieu dans lequel vous enseignez, en sachant que certaines stratégies proposées sont plus faciles à mettre en œuvre dans certains contextes (par exemple, celui du face à face pédagogique en terrain de stage) que dans d'autres (par exemple, celui du cours magistral réunissant plusieurs centaines d'étudiants).

\section{Agir positivement sur la perception de la valeur de l'activité}

Pour agir positivement sur cette perception, vous pourrez mettre en œuvre plusieurs stratégies.

\section{Explorez les attentes et les projets des étudiants}

Les étudiants valorisent d'autant plus une activité donnée que celle-ci est en adéquation avec leurs propres objectifs, leurs attentes et leurs projets ${ }^{[18,19]}$.
Il est donc important d'explorer et d' aider les étudiants à préciser ces derniers ${ }^{[1]}$. Plusieurs stratégies peuvent être déployées dans cette perspective. Il peut s'agir de réaliser un tour de table en début de formation, d'interroger les participants dans le cadre d'entretiens individuels ou collectifs, ou de leur administrer un questionnaire écrit. Dans le contexte du cours magistral, vous pourriez par exemple soumettre aux participants la question «Qu'attendez-vous de ce cours», et leur laisser un temps suffisant pour formaliser et noter par écrit leur réponse, avant de la verbaliser ${ }^{[20]}$. Dans la mesure du possible, il sera important de mettre en adéquation des objectifs d'apprentissage et les attentes et projets des étudiants, en établissant des liens. À défaut, il est utile d'expliquer aux étudiants pourquoi leurs attentes ne peuvent être comblées dans le contexte de la séquence d'apprentissage donnés.

\section{Expliquez à quoi servira la matière enseignée}

Le fait de prendre le temps de bien expliquer l'intérêt et l'utilité des contenus d'apprentissage visés par la formation, en exposant notamment les objectifs 
d'apprentissage, aide les étudiants à donner de la valeur aux activités ${ }^{[7,21]}$. Cette démarche est d'autant plus importante que les étudiants ont souvent des difficultés à identifier la valeur d'un cours, en particulier magistral, notamment lorsque celui-ci concerne les sciences biomédicales ${ }^{[2,20,22]}$. Les objectifs d'apprentissage devraient idéalement, et en tout cas le plus souvent possible, être mis en lien avec l'activité professionnelle future des étudiants, par exemple, en soulignant l'intérêt de telle ou telle notion abordée dans un cours d'anatomie, de physiologie ou d'embryologie pour la pratique médicale ${ }^{[1]}$. Concernant les sciences biomédicales, il y a également un intérêt, sur le plan motivationnel, à les intégrer dans une approche dite «orientée vers la clinique» et de favoriser précocement les contacts avec les patients $^{[22]}$, dans le cadre de ce que Dent \& Harden nomment une «intégration verticale» ${ }^{[23]}$.

\section{Associez la réalisation de l'activité à un défi}

Beaucoup d'enseignants pensent à tort que commencer une séquence d'enseignement avec des objectifs faciles à atteindre permet d'augmenter la motivation des étudiants à poursuivre leur apprentissage ${ }^{[7]}$. Or, des objectifs trop faciles à atteindre provoquent rapidement un désintérêt pour l'activité ${ }^{\text {[24] }}$; à l'inverse, des objectifs trop difficiles à atteindre peuvent conduire à l'abandon de l'activité. Afin d'associer les objectifs d'apprentissage à un impact positif sur la perception de la valeur de l'activité correspondante, ces activités devraient donc être associées à une forme de défi, c'est-à-dire qu'elles ne devraient être ni trop faciles ni trop difficiles à réaliser et qu'elles devraient, dans la mesure du possible, être associées à une disponibilité de l'assistance nécessaire pour que les étudiants réussissent ${ }^{[7,25]}$.

\section{Problématisez les apprentissages}

Les difficultés qu'éprouvent les étudiants à mettre en lien les contenus d'apprentissage ciblés en première année et l'exercice du métier de médecin sont à l'origine d'une diminution de leur motivation ${ }^{[2]}$. Le fait de problématiser une séquence d'apprentissage comme dans l'approche par problèmes - donne aux étudiants l'opportunité d'établir des liens entre les sciences biomédicales et les disciplines cliniques, en comprenant, ou même en résolvant des problèmes qu'ils rencontreront dans le cadre de leur activité professionnelle future. Cela majorera leur perception de la valeur de l'activité ${ }^{\text {26] }}$, dès lors que le problème est associé à un certain degré d'authenticité, c'est-à-dire que les étudiants considèrent qu'il ressemble aux problèmes qu'ils auront à résoudre dans l'exercice de leur future profession ${ }^{[7]}$. Ce problème peut prendre la forme d'une anecdote, de ressources iconographiques ou d'une vignette clinique décrivant, en début de cours, le cas d'un patient. Cette recherche d'authenticité du contexte d'apprentissage devrait également concerner les activités d'évaluation, auxquelles l'étudiant accordera dès lors une autre valeur que celle consistant uniquement à le sanctionner, puisqu'il prendra conscience du fait que les apprentissages qu'il a réalisés lui ont servi à résoudre des tâches en lien avec sa vie professionnelle future ${ }^{[7]}$.

\section{Renforcez les liens entre la théorie et la pratique}

Plusieurs études tendent à montrer l'influence des liens qu'est capable d'établir un professionnel de santé en formation initiale ou continue entre la théorie et la pratique, sur la perception de la valeur d'une activité d'apprentissage ${ }^{[21,27]}$. Ces liens sont plus faciles à établir chez les étudiants qui possèdent une connaissance préalable de l'activité clinique en lien avec la matière enseignée ${ }^{[27,28]}$. Ainsi, établir des liens entre les stages et les cours est une stratégie potentiellement intéressante sur le plan motivationnel ${ }^{[29]}$. Il est cependant important que vous considériez que beaucoup d'étudiants ne parviendront pas à établir eux-mêmes ces liens. Il est donc également de la responsabilité des enseignants de consacrer du temps, pendant leur cours, à rendre explicites les liens entre la matière enseignée et son utilisation en stage. Les stratégies exposées dans les précédents paragraphes doivent vous aider à atteindre cet objectif.

\section{Agir positivement sur le sentiment d'efficacité personnelle}

Un certain nombre de stratégies discutées afin d'agir positivement sur la perception de la valeur de 
l'activité présentent également un impact positif sur le sentiment d'efficacité personnelle. C'est notamment le cas de la problématisation des tâches d'apprentissage et du soin mis à les présenter selon un degré raisonnable d'authenticité ${ }^{[3,26]}$. Les autres stratégies sont décrites ci-dessous.

\section{Favorisez la réussite et promouvez les évaluations motivantes}

Les succès majorent le sentiment d'efficacité personnelle, alors que les échecs le déprécient ${ }^{[8,11]}$. Dans l'enseignement supérieur, la réussite et l'échec sont le plus souvent jugés par les étudiants par rapport à la note qu'ils obtiennent lors d'épreuves sommatives et certificatives. Il est donc important de ne pas se contenter de sanctionner les apprentissages par l'attribution d'une mauvaise note, mais avant tout de souligner les apprentissages réalisés et, par la suite, d'aider les étudiants à reconnaître leur échec. Cet accompagnement passe notamment par l'importance de clarifier et de rendre explicites auprès des étudiants les critères que vous utiliserez pour les évalue ${ }^{[7]}$. Il est également souhaitable d'associer dès que possible la note à des commentaires et de rassurer l'étudiant, dès lors que cela se révèle réaliste, sur le fait qu'il est capable de réussir ${ }^{[7]}$. Il s'agit enfin, dans le cadre des pratiques évaluatives, de valoriser d'autres dimensions des apprentissages réalisés que la seule réussite, par exemple, les stratégies d'apprentissage utilisées par l'étudiant, les progrès qu'il a réalisés, et sa persévérance $^{[3,7,25,30]}$.

Prenez en compte les conséquences de l'expérience vicariante

Selon Bandura, le fait d'observer une personne agir et réussir ou échouer dans la tâche qu'elle réalise est de nature à influencer la motivation des observateurs $^{[11]}$. «L'expérience vicariante» repose sur les inférences établies par les observateurs à partir de comparaisons sociales, qui sont à l'origine d'une forme d'autodiagnostic quant à ses propres capacités ${ }^{[11]}$. Vous devriez ainsi tenter d'en gérer les conséquences, dès lors que vous considérerez qu'une expérience d'apprentissage a pu produire des effets négatifs à travers ce mécanisme. Dans cette perspective, le rôle du feed-back - destiné tant à la personne impliquée dans la situation qu' aux observateurs - est déterminant.

\section{Offrez un feed-back bienveillant}

Un feed-back bienveillant ne peut reposer sur la seule note. La persuasion orale constitue l'un des leviers susceptibles de forger le sentiment d'efficacité personnelle chez un étudiant ${ }^{[11]}$. Afin d'en faire un facteur motivant, il est important d'inclure dans les feed-back les éléments positifs de la prestation d'un étudiant, tout en demeurant bien entendu réaliste par rapport à la prestation ${ }^{[11]}$. Cela nécessitera souvent un effort conscient, car les enseignants ont spontanément tendance à focaliser exclusivement le feed-back sur les erreurs commises par les étudiants. Un feed-back motivant est par ailleurs constructif et non menaçant, c'est-à-dire qu'il doit être ciblé sur des questions d'apprentissage et sur la façon d' aider l'étudiant dans les prochaines étapes de son apprentissage, et non sur la personne elle-même ${ }^{[25,31]}$. Cela impliquera parfois de juger non pas l'étudiant, mais le contexte dans lequel la tâche a été réalisée par celui-ci, ce qui permettra de normaliser certains échecs, par exemple lorsqu'ils surviennent précocement dans le cursus, au regard de tâches complexes ${ }^{[22]}$. Les mots utilisés ont également leur importance afin de ne pas influencer négativement le sentiment d'efficacité personnelle d'un étudiant. Par exemple, on préférera «axes d'amélioration» à «erreurs», afin de ne pas connoter négativement le feed-back ${ }^{[1]}$.

\section{Permettez aux étudiants d'endosser des fonctions d'enseignement}

Permettre aux étudiants d'endosser, à certaines occasions, des activités d'enseignement peut également avoir un impact positif sur leur sentiment d'efficacité personnelle. C'est notamment possible dans le cadre de l'apprentissage par problèmes, où les étudiants ont l'opportunité d'expliquer à leurs pairs les contenus dont ils ont acquis la maîtrise ${ }^{[22]}$. 
C'est également le cas dans des dispositifs de tutorat d'étudiants des années inférieures par des étudiants des années supérieures ${ }^{[22]}$.

\section{Agir positivement sur la perception de contrôlabilité}

La principale stratégie permettant d'agir positivement sur la perception de contrôlabilité des étudiants consiste à leur donner l'opportunité de faire des choix. Les choix offerts aux étudiants ne sont cependant pas tous motivants ${ }^{[32]}$. Les choix motivants sont ceux qui apparaissent pertinents, intéressants ou importants pour les apprenants - c'est-à-dire qui constitueront des opportunités d'auto accomplissement - tout en étant compatibles avec les capacités des étudiants et en leur offrant un encadrement qui les rassurera et favorisera des apprentissages significatifs ${ }^{[33]}$. Le curriculum des étudiants en sciences de la santé est ponctué de nombreuses opportunités de choix. Par exemple, ces étudiants sont souvent amenés à choisir un stage parmi d'autres ou des unités d'enseignement optionnelles. L'opportunité donnée aux étudiants en séance d'apprentissage par problèmes de formuler leurs propres objectifs d'apprentissage, de choisir, dans une certaine mesure, les tâches à accomplir au sein du groupe pour résoudre le problème posé et les sources d'information qu'ils consulteront dans cette perspective, est également de nature à majorer la perception de contrôlabilitée ${ }^{[22,26]}$. Les choix peuvent aussi concerner les modalités d'évaluation, par exemple, en laissant aux étudiants le choix des méthodes et des moments de l'évaluation $^{[22,34]}$.

\section{Conclusions}

La motivation est un déterminant majeur de l'apprentissage. Sa prise en compte par les enseignants du champ de la formation professionnelle en santé est parfois négligée. Ce guide a pour ambition d'outiller les enseignants avec un certain nombre de stratégies susceptibles d'être déployées en formation afin d'agir positivement sur la motivation des étudiants engagés dans les formations professionnelles en santé. Bien entendu, ces stratégies peuvent être transposées à d'autres milieux académiques d'enseignement des sciences de la santé, à d'autres milieux de l'éducation, et, plus généralement, à d'autres types d'activités humaines.

Bien que plusieurs stratégies exposées dans ce guide relèvent de stratégies générales d'enseignement, la motivation ne peut évidemment pas être considérée à elle seule comme un ressort suffisant de la qualité des apprentissages. Vous devrez donc associer le déploiement de ces stratégies à des approches, méthodes, techniques et outils relevant plus largement des pédagogies actives, héritées des courants cognitivistes et constructivistes de l'apprentissage.

\section{Déclaration d'intérêts}

Les auteurs ne déclarent pas de conflits d'intérêts en lien avec le contenu de cet article. Les auteurs sont seuls responsables du contenu de cet article.

\section{Approbation éthique}

\author{
Sans objet
}

\section{Contributeurs}

Thierry Pelaccia est maître de conférences des universités en médecine d'urgence. Il exerce la médecine d'urgence au sein du service d'aide médicale urgente et du service mobile d'urgence et de réanimation du Centre hospitalier universitaire de Strasbourg (France). Il dirige également le Centre de formation et de recherche en pédagogie des sciences de la santé (CFRPS), à la faculté de médecine de l'Université de Strasbourg.

Rolland Viau est professeur émérite à l'Université de Sherbrooke, au Canada. Depuis 30 ans, ses recherches et son enseignement portent sur la motivation à apprendre en contexte de formation.

Les deux auteurs ont co-rédigé solidairement le manuscrit. 


\section{Références}

1. Kusurkar RA, Ten Cate TJ, van Asperen M, Croiset G. Motivation as an independent and a dependent variable in medical education: a review of the literature. Med Teach 2011;33:e242-62.

2. Barker J, Olson J. Medical students' learning strategies: evaluation of first year changes. J Miss Acad Sci 1997;42:96-100.

3. Stegers-Jager KM, Cohen-Schotanus J, Themmen APN. Motivation, learning strategies, participation and medical school performance. Med Educ 2012;46:678-88.

4. Kusurkar RA, Ten Cate TJ, Vos CMP, Westers P, Croiset G. How motivation affects academic performance: a structural equation modelling analysis. Adv Health Sci Educ Theory Pract 2013;18:57-69.

5. Sobral DT. What kind of motivation drives medical students' learning quests? Med Educ 2004;38:950-7.

6. Wilson J. A two factor model of performance approach goals in student motivation for starting medical school. Issues Educ Res 2009;19:271-81.

7. Viau R. La motivation en contexte scolaire. Bruxelles : De Boeck, 2009.

8. Holland C. Critical review: medical students' motivation after failure. Adv Health Sci Educ Theory Pract 2016;21:695-710.

9. Graham S, Weiner B. Theories and principles of motivation. In : Berliner DC, Calfee RC (eds.). Handbook of Educational Psychology. New York: Macmillan, 1996:63-84.

10. Stipek D. Motivation to learn: from theory to practice. Boston: Allyn and Bacon, 2002.

11. Bandura A. Self-efficacy: the exercise of control. New York: W.H. Freeman \& Company, 1997.

12. Murphy PK, Alexander PA. A motivated exploration of motivation terminology. Contemp Educ Psychol 2000;25:3-53.

13. Kusurkar RA, Croiset G, Mann KV, Custers E, Ten Cate O. Have motivation theories guided the development and reform of medical education curricula? A review of the literature. Acad Med 2012;87:735-43.

14. Eccles JS, Wiglfield A, Schiefele U. Motivation to succeed. In : Damon W (ed.). Handbook of Child Psychology. New York: J. Wiley, 1998:1017-95.
15. Pintrich PR, De Groot V. Motivational and self-regulated learning components of classroom academic performance. J Educ Psychol 1990;82:33-40.

16. Pintrich PR, Schrauben B. Students' motivational beliefs and their cognitive engagement in classroom academic tasks. In : Schunk DH, Meece JL (eds.). Student perceptions in the classroom. Hillsdale, NJ: Lawrence Erlbaum Associates, 1992:149-83.

17. Ryan RM, Deci EL. Self-determination theory and the facilitation of intrinsic motivation, social development, and well-being. Am Psychol 2000;55:68-78.

18. Lens W, Decruyenaere M. Motivation and de-motivation in secondary education: Student characteristics. Learn Instr 1991;1:145-59.

19. Pelaccia T, Delplancq H, Triby E, Leman C, Bartier JC, Dupeyron J-P. La motivation en formation : une dimension réhabilitée dans un environnement d'apprentissage en mutation. Pédagogie Médicale 2008;9:103-21.

20. McKeachie W, Svinicki M. McKeachies' teaching tips: strategies, research, and theory for college and university teachers. Boston: Houghton Mifflin, 2013.

21. Hopstock LA. Motivation and adult learning: a survey among hospital personnel attending a CPR course. Resuscitation 2007;76:425-30.

22. Ten Cate TJ, Kusurkar RA, Williams GC. How selfdetermination theory can assist our understanding of the teaching and learning processes in medical education. AMEE guide No. 59. Med Teach 2011;33:961-73.

23. Dent J, Harden R. A practical guide for medical teachers. Ediburgh: Churchill Livingstone, 2001.

24. Paris S, Turner JC. Situated motivation. In: Pintrich, PR, Brown DR, Weinstein CE (eds.). Student motivation, cognition, and learning. Hillsdale, NJ: Lawrence Erlbaum Associates, 1994:213-37.

25. Kusurkar RA, Croiset G, Ten Cate TJ. Twelve tips to stimulate intrinsic motivation in students through autonomy-supportive classroom teaching derived from self-determination theory. Med Teach 2011;33:978-82.

26. Pedersen S. Motivational orientation in a problembased learning environment. J Interact Learn Res 2003;14:51-77.

27. Pelaccia T, Delplancq H, Triby E, Bartier J-C, Leman $\mathrm{C}$, Dupeyron J-P. Impact of training periods in the 
emergency department on the motivation of health care students to learn. Med Educ 2009;43:462-9.

28. Wigfield A, Eccles JS. Expectancy-value theory of achievement motivation. Contemp Educ Psychol 2000;25:68-81.

29. Cottin V, Mornex J-F, Cordier J-F. Enseignement magistral : intérêt potentiel de son intégration aux stages hospitaliers et de la réalisation de contrôles de connaissance impromptus. Pédagogie Médicale 2002;3:97-100.

30. Mann KV. Motivation in medical education: how theory can inform our practice. Acad Med 1999;74:237-9.
31. van de Ridder JMM, Stokking KM, McGaghie WC, ten Cate OTJ. What is feedback in clinical education? Med Educ 2008;42:189-97.

32. Flowerday T, Schraw G, Stevens J. The role of choice and interest in reader engagement. $\mathrm{J}$ Exp Educ 2004;72:93-114.

33. Katz I, Assor A. When choice motivates and when it does not. Educ Psychol Rev 2007;19:429.

34. Reeve J, Nix G, Hamm D. Testing models of the experience of self-determination in intrinsic motivation and the conundrum of choice. J Educ Psychol 2003;95:375-92. 